\title{
HOROBALLS IN SIMPLICES AND MINKOWSKI SPACES
}

\author{
A. KARLSSON, V. METZ, AND G. A. NOSKOV \\ Received 5 August 2005; Revised 23 July 2006; Accepted 25 July 2006
}

We obtain precise descriptions of all horoballs for Hilbert's geometry on simplices and for normed finite-dimensional vector spaces with norm given by some polyhedron. Certain geometrical consequences are deduced and several other applications are pointed out, which concern the dynamics of important classes of nonlinear self-maps of convex cones.

Copyright (c) 2006 Hindawi Publishing Corporation. All rights reserved.

\section{Introduction and results}

Our interest in horoballs comes on the one hand from the problem of describing the dynamics of certain important classes of self-maps of convex cones, and on the other hand from the work of Rieffel [17] on quantum metric spaces in the context of Connes' noncommutative geometry. Horofunctions also appeared recently in a general law of large numbers for random walks on groups [11].

As is remarked in [17, page 607], horofunctions seem not to have received much study previously. Most work so far has been devoted to spaces of nonpositive curvature (CAT(0)-spaces), see, for example, [1]. However, it is also true that Busemann functions or horofunctions have been an important tool in the study of Riemannian manifolds of nonnegative curvature.

Hilbert's geometry on convex sets and Minkowski's geometry on vector spaces (here meaning normed finite dimensional vector spaces over the reals) are not CAT(0), except in very special cases: hyperbolic geometry and Euclidean geometry, respectively. This makes these spaces interesting for further study of horofunctions outside the universe of nonpositive curvature. Indeed, Rieffel raises two questions [17, Questions 6.5, 6.6] about the horofunction compactification, Busemann functions, and the action of $V$ on its boundary for normed finite dimensional vector spaces $V$. In Section 4, we give some of the answers to these questions when the norm is given by a polyhedron (which is the case most opposite to Euclidean geometry). This will be in contrast to the well-known fact that if $X$ is a complete metric space satisfying the CAT(0)-condition or "nonpositive 
curvature," then the map from geodesic rays to horofunctions is a bijection. Indeed, the horofunction boundary is homeomorphic to the standard ray boundary [1].

Theorem 3.4 determines all the horoballs in Hilbert's metric $k$ on an open simplex $\Delta$ embedded in $\mathbb{R}^{d}$, see the figures in that section for a quick insight into how they look. Note that it is well known that $(\Delta, k)$ is isometrically isomorphic to a certain Minkowski space with polyhedral unit ball (see [16, Proposition 1.7], [6, Proposition 7], or [7]). Therefore, certain techniques from polyhedral Minkowski spaces can be used for $(\Delta, k)$. Unfortunately, the isometry is somewhat involved and not well suited for "translating" results to $(\Delta, k)$ directly.

In the same section, we also draw a few geometrical consequences from Theorem 3.4: every horoball is an intersection of certain basic ones, the horofunction compactification $\Delta_{\infty} \cup \Delta$ is explicitly given, and every geodesic ray converges in the Euclidean topology of $\mathbb{R}^{d}$.

Further applications outside of pure geometry, namely to dynamical systems, are discussed in Section 5.

\section{Preliminaries on horoballs and tangent cones}

Let $(X, d)$ be a metric space with unbounded metric $d$. We endow $C(X)$, the space of continuous real-valued functions on $X$, with the topology of uniform convergence on bounded sets. Fix a reference point $x_{0} \in X$ and consider the map $\Psi: X \rightarrow C(X)$ with

$$
\Psi: z \longmapsto d(z, \cdot)-d\left(z, x_{0}\right) .
$$

It continuously injects $X$ into $C(X)$ by the triangle inequality and positivity. A metric space is called proper if every closed bounded ball is compact. When this is the case for $(X, d)$, the Arzelá-Ascoli theorem implies that $\overline{\Psi(X)}$, the closure of $\Psi(X)$ in $C(X)$, is compact. Thus $\overline{\Psi(X)}$ is a compactification of $X$. Two compactifications with different reference points are homeomorphic because their functions only differ by additive constants. The boundary points are

$$
X_{\infty}:=\overline{\Psi(X)} \backslash \Psi(X)
$$

The elements of $X_{\infty}$ are called horofunctions. Rieffel calls $X_{\infty}$ the metric boundary. So a sequence $\left(z_{n}\right)_{n} \subset X$ going to infinity converges to $\xi \in X_{\infty}$ if and only if the sequence of functions $\left(d\left(z_{n}, \cdot\right)-d\left(z_{n}, x_{0}\right)\right)_{n} \subset C(X)$ converges uniformly on compact sets to some horofunction $h=h_{\xi}$. The lower level set $\left\{z: h_{\xi}(z) \leq C\right\}, C \in \mathbb{R}$, is termed a horoball and the level set a horosphere. We refer to [2] for further details.

At first glance this compactification looks quite abstract. But suppose $(X, d)$ is a geodesic space and let all geodesics be parameterized by arc length. Then geodesic rays always define a horofunction.

Example 2.1. Let $\gamma$ be a $d$-geodesic ray issuing from $\gamma(0)=: y \in X$. For $z \in X$, the limit of $d(\gamma(n), z)-d(\gamma(n), y)$ for $n \rightarrow \infty$ exists, since the sequence is monotonically decreasing in $n$ and bounded below by $-d(z, y)$, according to the triangle inequality. Thus it defines a horofunction and corresponding horoballs. 
Let us denote the closed $d$-ball of radius $r$ centered at $x \in X$ by $B(x, r)$ and the corresponding sphere by $S(x, r)$. For any sequence $\left(X_{n}\right)_{n}$ of subsets of $X$ define the metric upper limit by

$$
d \limsup X_{n}:=\bigcap_{n \geq 1} \overline{\bigcup_{k \geq 0} X_{n+k}} .
$$

That is $d \lim \sup X_{n}$ consists of all points $x$ for which there exists an increasing sequence $\left(n_{k}\right)_{k}$ and points $x_{n_{k}} \in X_{n_{k}}$ such that $\left(x_{n_{k}}\right)_{k}$ converges to $x$ in $(X, d)$. Some authors call this limit the Kuratowski-Painlevé upper limit [3].

Lemma 2.2. Let $(X, d)$ be a geodesic metric space with reference point $x_{0}$. Suppose that an unbounded sequence $\left(z_{n}\right)_{n} \subset X$ converges to a point $\xi \in X_{\infty}$. Denote the horofunction of $\xi$ by $h_{\xi}$. Then the horoball of level $C \in \mathbb{R}$ about $\xi$ looks as follows:

$$
B_{\xi}^{h}(C):=\left\{x \mid h_{\xi}(x) \leq C\right\}=d \lim \sup B\left(z_{n}, d\left(z_{n}, x_{0}\right)+C\right) .
$$

Proof. Suppose $h_{\xi}(x)=\lim _{n \rightarrow \infty}\left(d\left(z_{n}, x\right)-d\left(z_{n}, x_{0}\right)\right) \leq C$, then for any $\varepsilon>0$ the inclusion $x \in B\left(z_{n}, d\left(z_{n}, x_{0}\right)+C+\varepsilon\right)$ holds for all but finitely many $n$, hence $x$ belongs to the righthand side. Conversely, if $x$ belongs to the right-hand side, then there exists an increasing sequence $\left(n_{k}\right)_{k}$ and points $x_{n_{k}} \in B\left(z_{n_{k}}, d\left(z_{n_{k}}, x_{0}\right)+C\right)$ such that $\left(x_{n_{k}}\right)_{k}$ converges to $x$ in $(X, d)$. It follows that $d\left(z_{n_{k}}, x_{n_{k}}\right)-d\left(z_{n_{k}}, x_{0}\right) \leq C$. The triangle inequality shows that the limit of the left-hand side is $h_{\xi}(x)$.

Suppose $(X, d)$ is embedded in $\mathbb{R}^{N}$, which we endow with the Euclidean norm $\|\cdot\|$. Consider a Euclidean ray issuing from the point $y \in X$ and suppose it contains a $d$ geodesic ray $\gamma$. Then Example 2.1 tells us that $(\gamma(n))_{n}$ defines a horofunction and horoballs $B_{\gamma}^{h}(C)$. We use Lemma 2.2 to calculate these horoballs.

For any closed subset $M \subset \mathbb{R}^{N}$, we define the tangent cone $T(x, M)$ of $M$ at $x \in M$ by $v \in T(x, M)$ if and only if there exists $\left(v_{n}\right)_{n}$ converging to $v$ in $\left(\mathbb{R}^{N},\|\cdot\|\right)$ and $\left(\lambda_{n}\right)_{n} \subset$ $(0, \infty)$ converging to 0 such that $x+\lambda_{n} v_{n} \in M$ for all $n \in \mathbb{N}[9]$. With this definition the tangent cone is really a cone, that is, it is invariant under dilations. Furthermore, it is invariant under translations or dilations simultaneously of $M$ at $x$.

Lemma 2.3. Let $M \subseteq \mathbb{R}^{N}$ be convex and contain 0 . Then any two sequences $\left(\mu_{n}\right)_{n},\left(\lambda_{n}\right)_{n} \subset$ $(0, \infty)$ tending to $\infty$ satisfy

$$
d \limsup _{n \rightarrow \infty} \mu_{n} M=d \limsup _{n \rightarrow \infty} \lambda_{n} M
$$

Moreover, $T(0, M)=d \lim \sup _{n \rightarrow \infty} n M$.

Proof. By symmetry it is enough to show one inclusion. Suppose $\left(\mu_{n_{k}} v_{k}\right)_{k}$, with $v_{k} \in M$, tends to $v \in d \lim \sup _{n \rightarrow \infty} \mu_{n} M$. For every integer $k$ choose $l_{k}$ such that $\lambda_{l_{k}} \geq \mu_{n_{k}}$. Then

$$
\mu_{n_{k}} v_{k}=\lambda_{l_{k}} \frac{\mu_{n_{k}}}{\lambda_{l_{k}}} v_{k}=: \lambda_{l_{k}} w_{k}
$$

Since $M$ is convex and contains 0 , we have $w_{k} \in M$. Consequently, $v$ is an element of $d \lim \sup _{n \rightarrow \infty} \lambda_{n} M$. 
Suppose $v \in T(0, M)$. Then there exist $\left(v_{n}\right)_{n}$ and $\left(\lambda_{n}\right)_{n}$ converging to $v$ and 0 , respectively, such that $\lambda_{n} v_{n} \in M$ for all $n \in \mathbb{N}$. Hence $v_{n} \in\left(1 / \lambda_{n}\right) M$ and for $n \rightarrow \infty$,

$$
v \in d \limsup _{n \rightarrow \infty} \frac{1}{\lambda_{n}} M=d \limsup _{n \rightarrow \infty} n M .
$$

Conversely, if $v=\lim _{n \rightarrow \infty} n v_{n}$ for a sequence $\left(v_{n}\right)_{n} \subset M$, then $(1 / n)\left(n v_{n}\right)=v_{n} \in M$. Since $n v_{n}$ tends to $v$, we arrive at $v \in T(0, M)$.

\section{Simplices}

In this section, all topological notions will be with respect to $\left(\mathbb{R}^{d},\|\cdot\|\right), d \geq 1$, unless stated otherwise. We denote the closure by $\overline{(\cdot)}$, the interior by $(\cdot)^{\circ}$, and the boundary by $\partial(\cdot)$. Let $\mathbb{R}_{+}^{d}$ be the cone of all vectors with nonnegative entries and let $H$ be the hyperplane of all points in $\mathbb{R}^{d}$ whose components sum up to one. Then $\Delta:=\left(\mathbb{R}_{+}^{d}\right)^{\circ} \cap H$ is the open standard $(d-1)$-simplex. Hilbert used Klein's model of the hyperbolic plane to define a metric $k$ on any open, bounded, convex domain in $\mathbb{R}^{d}$ [8]. More precisely, for two points $x, y \in \Delta$, let $x^{\prime}, y^{\prime}$ be the intersection points of the line through $x$ and $y$ with $\partial \Delta$ such that the points appear in the order $x^{\prime}, x, y, y^{\prime}$. Now define $k$ to be the logarithm of the cross ratio

$$
k(x, y):=\ln \frac{\left\|x^{\prime}-y\right\| \cdot\left\|x-y^{\prime}\right\|}{\left\|x^{\prime}-x\right\| \cdot\left\|y-y^{\prime}\right\|}
$$

The corresponding geometry is studied in $[5,6]$. By definition the $k$-distance between two points of $\Delta$ tends to $+\infty$, when one of them tends to $\partial \Delta$.

3.1. Hilbert's projective metric on $\mathbb{R}_{+}^{d}$. A classical and elegant way to construct $k$-balls on $\Delta$ is to use its projective invariance as in [5, Section 18]. We will follow this approach in considering another (pseudo) distance, $k^{\prime}$, of Hilbert on the simplicial cone $\mathbb{R}_{+}^{d}$ whose restriction to $\Delta$ is $k$.

Birkhoff extended $k$ to a pseudo distance $k^{\prime}$ on cones in the following way [4]. For $x, y \in \mathbb{R}^{d}$ the partial order induced by $\mathbb{R}_{+}^{d}$ on $\mathbb{R}^{d}$ is given by $x \leq y$, if $y-x \in \mathbb{R}_{+}^{d}$. That is, $x_{i} \leq y_{i}$ for all $i \in D:=\{1, \ldots, d\}$. We use $\operatorname{supp}(x)$ to denote $\left\{i \in D \mid x_{i}>0\right\}$, the support of $x$. When $x, y \in \mathbb{R}_{+}^{d} \backslash\{0\}$ satisfy $\operatorname{supp}(x) \supseteq \operatorname{supp}(y)$, then we are able to define the greatest lower bound of the quotient $x / y$ by

$$
\begin{aligned}
\min (x / y): & =\max \{\alpha>0 \mid \alpha y \leq x\} \\
& =\min \left\{x_{i} / y_{i} \mid i \in \operatorname{supp}(y)\right\}>0 .
\end{aligned}
$$

When $\operatorname{supp}(x) \subseteq \operatorname{supp}(y)$, then the least upper bound of $x / y$ is

$$
\begin{aligned}
\max (x / y) & :=\min (y / x)^{-1} \\
& =\max \left\{x_{i} / y_{i} \mid i \in \operatorname{supp}(y)\right\}>0 .
\end{aligned}
$$


A. Karlsson et al. 5

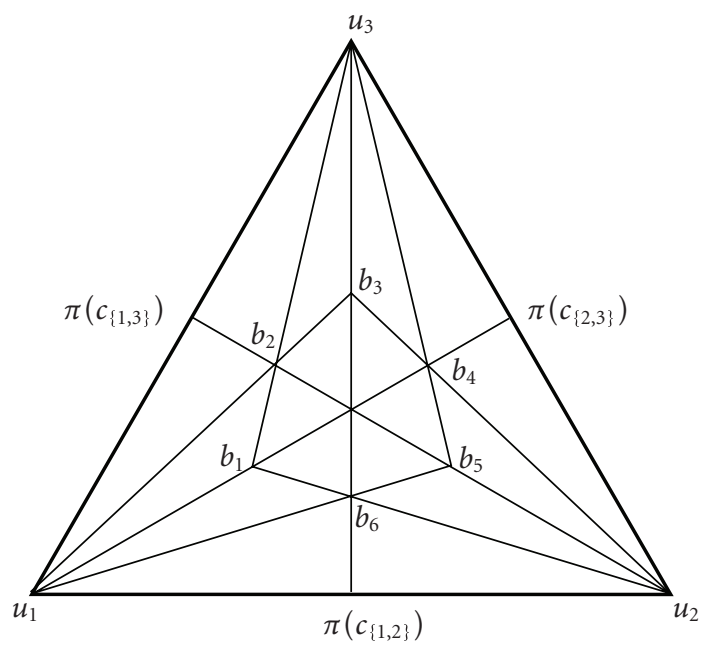

Figure 3.1. The $k$-ball in $\Delta$ centered at $c$ with extremal points $b_{1}, \ldots, b_{6}$.

When $\operatorname{supp}(x)=\operatorname{supp}(y)$, then Hilbert's projective metric $k^{\prime}$ is defined to be

$$
k^{\prime}(x, y):=\ln \frac{\max (x / y)}{\min (x / y)}
$$

The pseudometric $k^{\prime}$ is discussed in [16]. It satisfies $k^{\prime}(\alpha x, \beta y)=k^{\prime}(x, y)$ and it vanishes if and only if $x$ is a positive multiple of $y$. Thus the rays of $\left(\mathbb{R}_{+}^{d}\right)^{\circ}$ issuing from 0 are the equivalence classes of $k^{\prime}$. We project them to a point of $H$ with respect to the center 0 by $\pi: \mathbb{R}_{+}^{d} \backslash\{0\} \rightarrow \bar{\Delta}:$

$$
\pi(x):=\frac{x}{\|x\|_{1}}
$$

where $\|x\|_{1}:=\sum_{i}\left|x_{i}\right|$. Since $\|\cdot\|$ respects the $\mathbb{R}_{+}^{d}$-ordering, the metric space $\left(\Delta, k^{\prime}\right)$ is complete [16, Thmorem 1.2]. The $\|\cdot\|$ - and the $k^{\prime}$-distance are locally comparable on $\Delta$ [16, equations (1.21), (1.22)]. Therefore, $k^{\prime}$-closed balls are closed. Furthermore, $k^{\prime}$-balls are convex according to [16, Lemma 4.1]. But they might not be strictly convex as the $k^{\prime}$-ball of a 2 -simplex in Figure 3.1 shows.

Proposition 3.1 (see [14, equation (3.15)]). The two distances $k^{\prime}$ and $k$ coincide on $\Delta$.

For $i \in D$, let $u^{i} \in \mathbb{R}_{+}^{d}$ be the vector which is 1 in the $i$ th component and 0 elsewhere. For $x=\left(x_{1}, \ldots, x_{d}\right) \in \mathbb{R}^{d}$ and $I \subset D$, define

$$
x_{I}:=\sum_{i \in I} x_{i} u^{i}
$$

Analogously, $\Delta_{I}:=\left\{x_{I} \mid x \in \Delta\right\}$. When $y \in \mathbb{R}^{d}$ with $x \leq y$ we call $[x, y]:=\left\{z \in \mathbb{R}^{d} \mid x \leq\right.$ $z \leq y\}$ the cuboid of $x$ and $y$. For $r>0$ and $x \in \mathbb{R}_{+}^{d} \backslash\{0\}$, we define the closed $k$-ball of radius $r$ by $B(x, r):=\left\{y \in \mathbb{R}_{+}^{d} \mid k^{\prime}(x, y) \leq r\right\}$. This ball is actually a cone. 
Proposition 3.2. For $r>0$ and $x \in \Delta$, the extremal rays of the cone $B(x, r)$ are spanned by those extremal points of $\left[x, e^{r} x\right]$ which are not multiples of $x$. Especially, $B(x, r) \cap \Delta$ is a closed convex set with the $2^{d}-2$ extremal points

$$
\left\{\frac{x+\left(e^{r}-1\right) x_{I}}{1+\left(e^{r}-1\right)\left\|x_{I}\right\|_{1}} \mid \varnothing \subsetneq I \subsetneq D\right\} .
$$

Proof. Let $y \in \mathbb{R}_{+}^{d}$ be such that $k^{\prime}(x, y)=r$. By (3.4) this implies $\operatorname{supp}(y)=\operatorname{supp}(x)$ and the existence of an $\alpha>0$ such that $\alpha x \leq y \leq e^{r} \alpha x$. For each inequality, there is at least one coordinate in which equality holds. This shows $y / \alpha \in \partial\left[x, e^{r} x\right]$. The inclusion $\left[x, e^{r} x\right] \subseteq$ $B(x, r)$ follows from the definition of $k$. Thus $\pi(B(x, r))=\pi\left(\left[x, e^{r} x\right]\right)$.

For $I \subseteq D$, the extremal points of $\left[x, e^{r} x\right]$ are

$$
E(I):=E^{r}(I):=x+\left(e^{r}-1\right) x_{I} .
$$

Since $\pi$ maps nonzero linear combinations over $\mathbb{R}_{+}$to convex combinations, $\pi\left(\left[x, e^{r} x\right]\right)$ is convex. Its extremal points are contained in $\{\pi(E(I)) \mid I \subseteq D\}$. Since $\pi(E(D))=\pi(E(\varnothing))=$ $\pi(x)$, the set $\pi\left(\left[x, e^{r} x\right]\right)$ is the convex hull of $\{\pi(E(I)) \mid \varnothing \subsetneq I \subsetneq D\}$.

To show the extremality of $\{\pi(E(I)) \mid \varnothing \subsetneq I \subsetneq D\}$, we suppose that $y \in \partial B(x, r)$ is a linear combination $\sum_{i=1}^{n} \lambda_{i} z^{i}$ of points $z^{i} \in B(x, r)$ with $\lambda_{i}>0$, for $1 \leq i \leq n$. Use (3.2) to express $e^{k(x, y)}$ as the maximum over $(j, l) \in D^{2}$ of

$$
\frac{y_{j} x_{l}}{x_{j} y_{l}}=\frac{x_{l}}{x_{j}} \cdot \frac{\sum_{p=1}^{n} \lambda_{p} z_{j}^{p}}{\sum_{i=1}^{n} \lambda_{i} z_{l}^{i}}=\frac{x_{l}}{x_{j}} \cdot \sum_{p=1}^{n} \frac{z_{j}^{p}}{z_{l}^{p}}\left(\frac{\lambda_{p} z_{l}^{p}}{\sum_{i=1}^{n} \lambda_{i} z_{l}^{i}}\right) .
$$

Thus $y_{j} x_{l} / x_{j} y_{l}=e^{r}$ if and only if $z_{j}^{p} x_{l} / x_{j} z_{l}^{p}=e^{r}$ for all $1 \leq p \leq n$. Indeed, the quotient in (3.9) on the very left is known to be maximal, that is, $e^{r}$. The sum on the very right of the equation is a convex combination of quotients $x_{l} z_{j}^{p} / x_{j} z_{l}^{p}$ with positive weights given by the expression in brackets. Every $x_{l} z_{j}^{p} / x_{j} z_{l}^{p}$ is not bigger than $e^{r}$ because these quotients appear in measuring $k^{\prime}\left(x, z^{p}\right)$ with $z^{p} \in B(x, r)$. So the convex combination is maximal if and only if every $x_{l} z_{j}^{p} / x_{j} z_{l}^{p}$ is maximal, that is, $e^{r}$.

For $y=E(I)$, obviously $y_{j} x_{l} / x_{j} y_{l}=e^{r}$ for all $j \in D \backslash I$ and $l \in I$. So $\left\{z_{i}^{p} / x_{i} \mid i \in D\right\}$ consists only of two values whose quotient is $e^{r}$. Thus $z^{p}$ is a multiple of $y$.

Proposition 3.2 tells us that $k$-balls in $\Delta$ are $\pi$-images of cuboids. In other words, they are $(d-1)$-dimensional polytopes with $\left(2^{d}-2\right)$ extremal points. The latter fact is well known [6]. The hexagonal shape of a 2-dimensional ball is depicted in Figure 3.1. It is centered at $c:=\pi((1, \ldots, 1))$ and has the extremal points

$$
\begin{array}{rlrl}
b^{1} & :=\pi(E(\{1\})), & b^{2}:=\pi(E(\{1,3\})), & b^{3}:=\pi(E(\{3\})), \\
b^{4}:=\pi(E(\{2,3\})), & b^{5}:=\pi(E(\{2\})), & b^{6}:=\pi(E(\{1,2\})) .
\end{array}
$$

To understand the remaining lines in the figure we have to investigate the faces of a $k$-ball. 
A $k$-ball in $\Delta$ with a positive radius has dimension $d-1$. We want to know its $(d-2)$ dimensional faces. Let us call such a face a facet. Proposition 3.2 suggests trying the $\pi$ images of $(d-2)$-dimensional faces of $\left[x, e^{r} x\right]$, provided they do not contain $x$ or $e^{r} x$.

Corollary 3.3. The $k$-ball in $\Delta$, centered at $x \in \Delta$ with radius $r>0$, has $d(d-1)$ facets. For each pair of distinct indices $1 \leq i, j \leq d$, the facet is given by the extremal points $\{\pi(E(I)) \mid$ $\varnothing \subsetneq I \subsetneq D, i \in I \supsetneqq j\}$. Every other face of the $k$-ball is the intersection of a finite number of these facets.

Proof. According to (3.9), the convex sets in question define convex subsets of $\partial B(x, r)$, because they have the joint maximal quotient $\pi\left(E(I)_{i}\right) / \pi\left(x_{i}\right)$ and the joint minimal quotient $\pi\left(E(I)_{j}\right) / \pi\left(x_{j}\right)$. Every such set is by definition the $\pi$-image of a suitable $(d-2)$ dimensional face of $\left[x, e^{r} x\right]$. The faces of $\left[x, e^{r} x\right]$ lie in affine hyperplanes which do not contain 0 . Hence $\pi$ does not decrease their dimensions. Thus the convex sets in question have dimension $d-2$. For every point $b$ in a $k$-sphere, the quotients $\left\{b_{i} / x_{i} \mid i \in D\right\}$ have at least one maximal element and one minimal.

Varying the radius $r>0$ of the $k$-ball $B(x, r)$ in Proposition 3.2, we see that $\pi\left(E^{r}(I)\right)$ describes a line segment from $x$ to $\pi\left(x_{I}\right) \in \partial \Delta$. For every pair of distinct indices $1 \leq i$, $j \leq d$ the convex hull of $x$ and $\left\{\pi\left(x_{I}\right) \mid \varnothing \subsetneq I \subsetneq D, i \in I \not j\right\}$ is called an $x$-sector of $\Delta$. According to Corollary 3.3, the intersection of such an $x$-sector with a $k$-sphere centered at $x$ is a facet.

Figure 3.1 indicates the supporting hyperplanes of the 2 -dimensional $k$-ball centered at $c$. They are of course lines and their location is indicated by the line segments from $u^{i}$ to some $b^{j}, 1 \leq j \leq 6$, but not passing through $c$.

Let $b \in \partial \Delta$, that is, $b$ has a zero in at least one and in at most $d-1$ coordinates. For $\left(g^{n}\right)_{n} \subset \Delta$ and converging to $b,(3.2)$ shows

$$
\lim _{n \rightarrow \infty} \max \left(g^{n} / x\right)=\max (b / x) \in(0, \infty)
$$

3.2. Horoballs. The straight line segment between two different points of $\Delta$ is a $k$-geodesic [16, Propostion 1.9]. So $(\Delta, k)$ is a geodesic space. But $k$-geodesics are not necessarily unique because $k$-balls have faces as we have seen. A $k$-geodesic $g:[0, \infty) \rightarrow \Delta$ is called a $k$-geodesic ray. Thus Euclidean rays issuing from a point in $\Delta$ are $k$-geodesic rays. On $\Delta$ the $\|\cdot\|$ - and the $k$-topology coincide locally as we have seen already. Hence $k$-closed balls are $k$-compact, that is, $(\Delta, k)$ is a proper metric space. Now we can apply the results of Section 2 to find horofunctions and horoballs.

Let $\left(g^{n}\right)_{n} \subseteq \Delta$ converge to $b \in \partial \Delta$ and fix $x \in \Delta$. For notational convenience set $r(n):=$ $k\left(x, g^{n}\right)$ and $E^{r(n)}(I)=: E_{n}(I)$. We want to find a horoball defined by $\left(g^{n}\right)_{n}$ which contains $x$ in its boundary. We call such a horoball centered at $b$. That is, we have to consider a sequence of $k$-balls $B\left(g^{n}, r(n)\right), n \in \mathbb{N}$, which will hopefully converge (in the KuratowskiPainlevé distance) to a horoball centered at $b$. In our case, all $k$-balls are isomorphic polytopes, that is, they have the same face lattice described in Corollary 3.3. Their extremal points are $\left\{E_{n}(I) \mid \varnothing \subsetneq I \subsetneq D\right\}$. Suppose $\left(E_{n}(I)\right)_{n}$ converges to $E_{\infty}(I)$ for all $\varnothing \subsetneq I \subsetneq D$. Then the limiting set is the convex hull of $\left\{E_{\infty}(I) \mid \varnothing \subsetneq I \subsetneq D\right\}$ and the extremal points of the limiting set are contained in this set of limit points. 
According to Proposition 3.2, $E_{n}(I)$ is given, for $\varnothing \subsetneq I \subsetneq D$, by

$$
\begin{aligned}
y^{I}(n) & =\frac{g_{D \backslash I}^{n}+e^{r(n)} g_{I}^{n}}{\left\|g_{D \backslash I}^{n}\right\|_{1}+e^{r(n)}\left\|g_{I}^{n}\right\|_{1}} \\
& =\pi\left(g_{D \backslash I}^{n}\right)\left(1+\frac{e^{r(n)}\left\|g_{I}^{n}\right\|_{1}}{\left\|g_{D \backslash I}^{n}\right\|_{1}}\right)^{-1}+\pi\left(g_{I}^{n}\right)\left(1+\frac{\left\|g_{D \backslash I}^{n}\right\|_{1}}{e^{r(n)}\left\|g_{I}^{n}\right\|_{1}}\right)^{-1} .
\end{aligned}
$$

Let us look again at Figure 3.1. We replace $c$ by $g^{n}$ and move it towards $b \in \partial \Delta$, for example $b=\left(u^{1}+u^{2}\right) / 2$. The boundary of $B\left(g^{n}, r(n)\right)$ then contains the fixed point $x$, say $x=b^{3}$. Since $b^{1}(n), b^{5}(n), b^{6}(n)$ in the figure lie on rays emanating from $u^{1}$ or $u^{2}$, the sequences must end up on the line segment from $u^{1}$ to $u^{2}$. This is the first case we will consider.

Suppose $\operatorname{supp}(b) \cap I \neq \varnothing$, then $g_{I}^{n}$ tends to $b_{I} \neq 0$ for $n \rightarrow \infty$ and

$$
\liminf _{n \rightarrow \infty} \frac{\left\|g_{I}^{n}\right\|_{1}}{\left\|g_{D \backslash I}^{n}\right\|_{1}} \geq\left\|b_{I}\right\|_{1}>0 .
$$

Now (3.12) and the fact that $r(n)$ tends to $\infty$ for $n \rightarrow \infty$ imply

$$
E_{\infty}(I)=\pi\left(b_{I}\right)
$$

for $\varnothing \subsetneq I \subsetneq D$ with $\operatorname{supp}(b) \cap I \neq \varnothing$. All these points have their support in $\operatorname{supp}(b)$. They comprise $\left\{u^{i} \mid i \in \operatorname{supp}(b)\right\}$, which are the extremal points of $\overline{\Delta_{\operatorname{supp}(b)}}$. Hence the latter points are extremal in any horoball.

To motivate the case $\operatorname{supp}(b) \cap I=\varnothing$ geometrically, we look again at Figure 3.1. Let $b=u^{1}$ and look at $b^{4}(n)$ when $g^{n}$ moves towards $b$ for $n \rightarrow \infty$ and choose again $x:=b^{3}$. When $\left(\pi\left(g_{\{2,3\}}^{n}\right)\right)_{n}$ converges we can draw the limiting boundary of $B\left(g^{n}, r(n)\right)$. This is the type of problem we have to treat now.

Suppose $\operatorname{supp}(b) \cap I=\varnothing$. Then $g_{D \backslash I}^{n}$ tends to $b$ for $n \rightarrow \infty$. According to (3.12), we have to find the limits of $\pi\left(g_{I}^{n}\right)$ and $e^{r(n)}\left\|g_{I}^{n}\right\|_{1}$. To this end use (3.2) to deduce

$$
e^{r(n)} \cdot\left\|g_{I}^{n}\right\|_{1}=\frac{\max \left(g^{n} / x\right)}{\min \left\{\left(g_{j}^{n} /\left\|g_{I}^{n}\right\|_{1}\right)\left(1 / x_{j}\right) \mid j \in D\right\}} .
$$

By (3.11) the numerator converges to $\max (b / x)$. The denominator is

$$
\mu_{I}(n):=\min \left\{\frac{g_{j}^{n}}{\left\|g_{I}^{n}\right\|_{1}} \frac{1}{x_{j}} \mid j \in D\right\} .
$$

Obviously, $0<\mu_{I}(n) \leq \min _{i \in I}\left(1 / x_{i}\right)$. When $\left(\mu_{I}(n)\right)_{n}$ converges to $\mu_{I}$ and $\left(\pi\left(g_{I}^{n}\right)\right)_{n}$ converges to $\pi\left(g_{I}\right)$ for $n \rightarrow \infty$, then (3.12) implies

$$
E_{\infty}(I)=\frac{\mu_{I}}{\mu_{I}+\max (b / x)} b+\frac{\max (b / x)}{\mu_{I}+\max (b / x)} \pi\left(g_{I}\right) .
$$

To see when $\left(\mu_{I}(n)\right)_{n}$ converges to 0 we have to know the "speed" at which $\left(g_{i}^{n}\right)_{n}$ approaches 0 . For $i, j \in D$, we say that $i$ is faster than $j$ if $\limsup _{n \in \mathbb{N}}\left(g_{i}^{n} / g_{j}^{n}\right)=0$. Whenever 
there exists a $j \in D$ which is faster than some $i \in I$, then $\mu_{I}(n)$ converges to 0 for $n \rightarrow \infty$. Let $F$ be the set of all fastest components in $D$. Then $\mu_{I}(n)$ converges to 0 if and only if $I \nsubseteq F$. Consequently, (3.17) implies

$$
E_{\infty}(I)=\pi\left(g_{I}\right)
$$

for $\varnothing \subsetneq I \subseteq D \backslash \operatorname{supp}(b)$ with $I \nsubseteq F$.

To see when $\left(\mu_{I}(n)\right)_{n}$ converges to a positive value, we have to consider the only remaining cases $\varnothing \subsetneq I \subseteq F$. By the definition of $F$ we have for large enough $n$,

$$
\mu_{I}(n)=\min \left\{\frac{g_{j}^{n}}{\left\|g_{I}^{n}\right\|_{1}} \frac{1}{x_{j}} \mid j \in F\right\} .
$$

Using $g^{n}=\left\|g_{D \backslash F}^{n}\right\|_{1} \cdot \pi\left(g_{D \backslash F}^{n}\right)+\left\|g_{F}^{n}\right\|_{1} \cdot \pi\left(g_{F}^{n}\right)$ and $j \in F$, we derive

$$
\frac{g_{j}^{n}}{\left\|g_{I}^{n}\right\|_{1}}=\frac{\left\|g_{F}^{n}\right\|_{1} \cdot \pi\left(g_{F}^{n}\right)_{j}}{\|\| g_{F}^{n}\left\|_{1} \cdot \pi\left(g_{F}^{n}\right)_{I}\right\|_{1}}=\frac{\pi\left(g_{F}^{n}\right)_{j}}{\left\|\pi\left(g_{F}^{n}\right)_{I}\right\|_{1}} .
$$

Thus again for big $n$,

$$
\mu_{I}(n)=\min \left\{\frac{\pi\left(g_{F}^{n}\right)_{j}}{\left\|\pi\left(g_{F}^{n}\right)_{I}\right\|_{1}} \frac{1}{x_{j}} \mid j \in F\right\} .
$$

Theorem 3.4. Let $\left(g^{n}\right)_{n} \subseteq \Delta$ be a sequence converging to $b \in \partial \Delta$. Define $F$ to be the set of fastest components of $\left(g^{n}\right)_{n}$. The sequence $\left(g^{n}\right)_{n}$ defines a horoball if and only if $\left(\pi\left(g_{F}^{n}\right)\right)_{n}$ converges to a point $\pi\left(g_{F}\right)$, the "direction" of $\left(g^{n}\right)_{n}$. The extremal points of the horoball are given by

(i) every $u^{i}$ for $i \in D \backslash F$;

(ii) every $E_{\infty}(I)$ of (3.17) for $\varnothing \subsetneq I \subseteq F$, where

$$
\mu_{I}=\frac{\min \left(\pi\left(g_{F}\right) / x_{F}\right)}{\left\|\pi\left(g_{F}\right)_{I}\right\|_{1}} .
$$

Proof. Suppose $\left(\pi\left(g_{F}^{n}\right)\right)_{n}$ converges to $\pi\left(g_{F}\right)$. Then (3.14) and (3.18) show that $\pi\left(g_{I}\right)$ is possibly extremal for $\varnothing \subsetneq I \subseteq D \backslash F$. They comprise the points in (i).

When $\left(\pi\left(g_{F}^{n}\right)\right)_{n}$ converges, then $\pi\left(g_{F}\right) \in \overline{\Delta_{F}} \backslash \Delta_{F}$ implies that there are elements in $F$ which are faster than other elements of $F$. This is impossible by the definition of $F$. Thus $\pi\left(g_{F}\right) \in \Delta_{F}$ and $\pi\left(g_{F}\right)_{j}>0$ for all $j \in F$. Because of (3.21), $\mu_{I}>0$ has the formula given in (ii) and the corresponding points in (3.17) are possibly extremal.

Let $K$ be the convex hull of all points in (i) and (ii). All points of (i) are extremal in $\bar{\Delta}$ and thus in $K$. Fix $\varnothing \subsetneq I \subseteq F$. Then the corresponding point of (ii) has the formula (3.17) with $0<\mu_{I}<\infty$. Write $E_{\infty}(I)$ as a convex combination of the points in (i) and (ii) and consider the $I$-coordinates of $E_{\infty}(I)$. Formula (3.17) shows that points of (i) cannot contribute to these coordinates. By (ii) a contribution can only come from $E_{\infty}(J)$ with $\varnothing \subsetneq J \subseteq F$. Thus we only have to check $\varnothing \subsetneq J \subsetneq I$. The function $f(\mu):=$ $\max (b / x) /(\max (b / x)+\mu)$ is strictly decreasing in $\mu$ as long as $\max (b / x)>0$. The latter is 


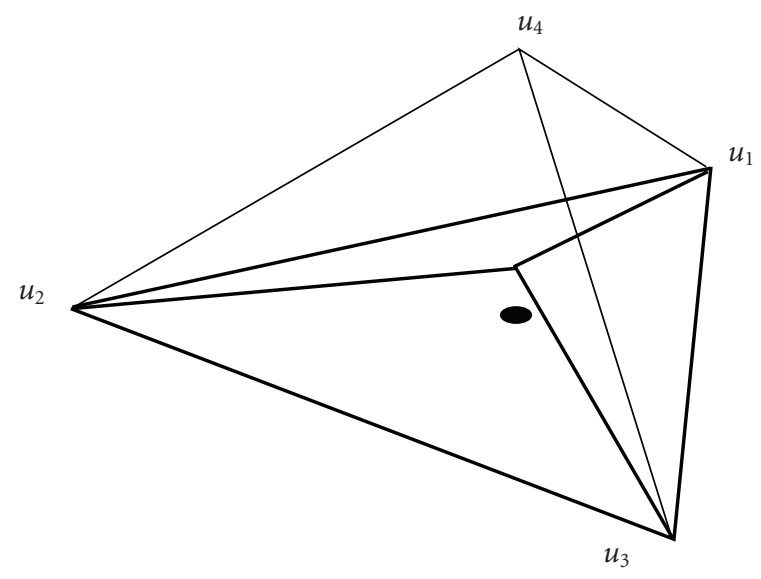

Figure 3.2. A horoball centered at $\left(u_{1}+u_{2}+u_{3}\right) / 3$.

true, since $x \in \Delta$ and $b \neq 0$. We know that $\pi\left(g_{F}\right)$ has full support $F$. Thus the formula in (ii) shows $\mu_{J}>\mu_{I}$, for $\varnothing \subsetneq J \subsetneq I$, which implies $f\left(\mu_{J}\right)<f\left(\mu_{I}\right)$. Hence a convex combination of all $f\left(\mu_{J}\right)$, for all $J$ as above, is always strictly less than $f\left(\mu_{I}\right)$. This shows that $E_{\infty}(I)$ is extremal.

Now suppose $\left(\pi\left(g_{F}^{n}\right)\right)_{n}$ diverges. Then it has accumulation points in $\overline{\Delta_{F}}$ and every convergent subsequence defines a horoball via the corresponding subsequence of $\left(g^{n}\right)_{n}$ according to our previous arguments. Take two accumulation points $a^{1} \neq a^{2}$ and their corresponding subsequences. When $\operatorname{supp}\left(a^{1}\right) \neq \operatorname{supp}\left(a^{2}\right)$, then (i) shows that their horoballs are different. When $\operatorname{supp}\left(a^{1}\right)=\operatorname{supp}\left(a^{2}\right)$, then (ii) and (3.17) imply that the $\operatorname{supp}(b)$ components of their $E_{\infty}(F)$-s coincide if and only if their $\mu_{F}$-s are the same. In this case their $E_{\infty}(F)$-s coincide if and only if $a^{1}=a^{2}$, which is false. Thus the horoballs differ when $a^{1} \neq a^{2}$. But when the horoballs are different, then the locally uniform convergence of $\left(\Psi\left(g^{n}\right)\right)_{n}$ is violated. Therefore, no horofunction exists and consequently no horoball for $\left(g^{n}\right)_{n}$.

We note that no directional assumptions on the convergence of $\left(g^{n}\right)_{n}$ to $b$ have to be made in Theorem 3.4 when $\operatorname{supp}(b)$ contains all but one component. A horoball of this type is depicted in Figure 3.2.

According to Theorem 3.4, there exist sequences $\left(g^{n}\right)_{n} \subset \Delta$ converging to $b \in \partial \Delta$ which define a horoball but $\left(\pi\left(g_{D \backslash \operatorname{supp}(b)}^{n}\right)\right)_{n}$ diverges in $\left(\mathbb{R}^{d},\|\cdot\|\right)$. Look for example at the right horoball of Figure 3.3 and imagine two different accumulation points in $\Delta_{\{2,3\}}$. The corresponding subsequences define the same horoball, because the extremal point of statement (ii) only depends on the support of the accumulation points. This shows clearly that the "direction" mentioned in Theorem 3.4 has to be a point with support $F$ but not the bigger support $D \backslash \operatorname{supp}(b)$.

It remains to check whether there exist divergent sequences defining a horoball. To this end we will refine the argument in the last paragraph of the proof of Theorem 3.4. 

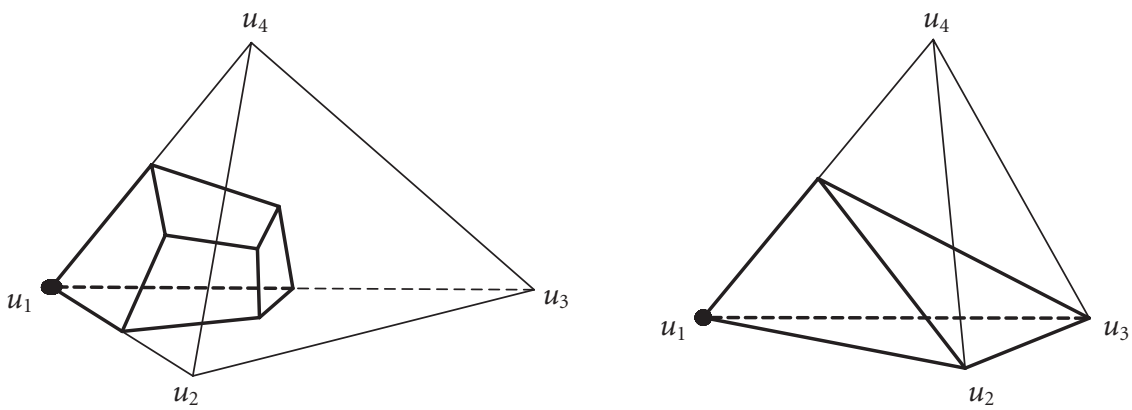

Figure 3.3. Two horoballs centered at $u_{1}$ with $\pi\left(g_{\{2,3,4\}}\right)$ equal to $\left(u_{2}+u_{3}+u_{4}\right) / 3$ on the left and $\left(u_{2}+\right.$ $\left.u_{3}\right) / 2$ on the right.

Corollary 3.5. Every sequence $\left(g^{n}\right)_{n} \subseteq \Delta$ accumulating only at $\partial \Delta$ that defines a $k$-horoball has to converge.

Proof. Suppose we are given a sequence $\left(g^{n}\right)_{n} \subseteq \Delta$ accumulating only at $\partial \Delta$ but possessing at least two accumulation points $a^{1} \neq a^{2}$. Each of them is the limit of a suitable subsequence of $\left(g^{n}\right)_{n}$. These subsequences must define the same horoball because otherwise the locally uniform convergence of $\left(\Psi\left(g^{n}\right)\right)_{n}$ is violated. Thus Theorem 3.4(i) shows that both sequences have the same set $F$ of fastest components. Furthermore, they must define the same $E_{\infty}(F)$ in statement (ii) of the same theorem to ensure coinciding horoballs. Let $y^{F}$ be an accumulation point of the sequence $\left(y^{F}(n)\right)_{n}$ defined in (3.12). Now (3.17) tells us that $\operatorname{supp}\left(y^{F}\right)$ is the disjoint union of $\operatorname{supp}\left(a^{i}\right)$ and $F$. So $\operatorname{supp}\left(a^{1}\right)$ coincides with supp $\left(a^{2}\right)$. Looking at the $F$-components of $E_{\infty}(F)$, we conclude that $\max \left(a^{1} / x\right)=\max \left(a^{2} / x\right)$. Considering now the $\operatorname{supp}\left(a^{1}\right)$-components of $E_{\infty}(F)$, we derive $a^{1}=a^{2}$.

3.3. Geometrical consequences. We will collect some geometrical information drawn from Theorem 3.4 .

We will see in the next corollary that every horoball centered at $b$ is the intersection of specific basic horoballs. Such a basic horoball is characterized by a center $b=u^{j}$, a single fastest component $F=\{k\}$, and a point $x \in \Delta$ in its boundary, for two different indices $j, k \in D$. Because of $F=\{k\}$, we have $\mu_{\{k\}}=1 / x_{k}$ by Theorem 3.4(ii) and

$$
E_{\infty}(\{k\}):=\frac{x_{j}}{x_{j}+x_{k}} u^{j}+\frac{x_{k}}{x_{j}+x_{k}} u^{k}
$$

according to (3.17). A horoball of this type is shown on the right-hand side of Figure 3.3. Corollary 3.6. Every closed horoball centered at $b$ with fastest components $F$ is the intersection of $|\operatorname{supp}(b)| \cdot|F|$ closed horoballs which are centered at a point of $\left\{u^{j} \mid j \in \operatorname{supp}(b)\right\}$, have a fastest component $\{i\}$, for some $i \in F$, and contain the extremal point $E_{\infty}(F)$ of the original ball. 
Proof. We have to show that the intersection of all basic horoballs in question has the same extremal points as the original horoball. By definition the intersection contains the extremal points $u^{i}, i \in \operatorname{supp}(b)$. So it remains to show that the extremal points of the original horoball which appear in Theorem 3.4(ii) are the missing extremal points of the intersection. This follows from

$$
\pi\left(E_{\infty}(F)_{\operatorname{supp}\left(E_{\infty}(I)\right)}\right)=E_{\infty}(I) \quad(\varnothing \subsetneq I \subseteq F) .
$$

To prove this we collect some remarks. The support of $E_{\infty}(I)$ is just $\operatorname{supp}(b) \cup I$, according to (3.17). Theorem 3.4(ii) shows that $\mu_{F}$ equals $\min \left(\pi\left(g_{F}\right) / x_{F}\right)$ and therefore $\mu_{I} \cdot\left\|\pi\left(g_{F}\right)_{I}\right\|_{1}=\mu_{F}$. By definition and the choice of $I$,

$$
\left\|\pi\left(g_{F}\right)_{I}\right\|_{1}=\left\|\frac{g_{F \cap I}}{\left\|g_{F}\right\|_{1}}\right\|_{1}=\frac{\left\|g_{I}\right\|_{1}}{\left\|g_{F}\right\|_{1}}
$$

Using (3.17) and these remarks, we see that $E_{\infty}(F)_{\operatorname{supp}(b) \cup I}$ equals

$$
\begin{aligned}
& \frac{\mu_{F}}{\mu_{F}+\max (b / x)} b+\frac{\max (b / x)}{\max (b / x)+\mu_{F}} \pi\left(g_{F}\right)_{I} \\
& =\frac{\mu_{I}\left\|\pi\left(g_{F}\right)_{I}\right\|_{1}}{\mu_{I}\left\|\pi\left(g_{F}\right)_{I}\right\|_{1}+\max (b / x)} b+\frac{\max (b / x)}{\max (b / x)+\mu_{I}\left\|\pi\left(g_{F}\right)_{I}\right\|_{1}} \cdot \frac{g_{I}}{\left\|g_{I}\right\|_{1}} \cdot \frac{\left\|g_{I}\right\|_{1}}{\left\|g_{F}\right\|_{1}} \\
& =\frac{\mu_{I}\left\|\pi\left(g_{F}\right)_{I}\right\|_{1}}{\mu_{I}\left\|\pi\left(g_{F}\right)_{I}\right\|_{1}+\max (b / x)} b+\frac{\max (b / x)\left\|\pi\left(g_{F}\right)_{I}\right\|_{1}}{\max (b / x)+\mu_{I}\left\|\pi\left(g_{F}\right)_{I}\right\|_{1}} \pi\left(g_{I}\right) \text {. }
\end{aligned}
$$

Because of $b, \pi\left(g_{I}\right) \in \bar{\Delta}$, we derive

$$
\left\|E_{\infty}(F)_{\operatorname{supp}(b) \cup I}\right\|_{1}=\frac{\left(\mu_{I}+\max (b / x)\right)\left\|\pi\left(g_{F}\right)_{I}\right\|_{1}}{\mu_{I}\left\|\pi\left(g_{F}\right)_{I}\right\|_{1}+\max (b / x)} .
$$

Together we deduce

$$
E_{\infty}(F)_{\operatorname{supp}(b) \cup I}=E_{\infty}(I) \cdot\left\|E_{\infty}(F)_{\operatorname{supp}(b) \cup I}\right\|_{1} .
$$

A consequence of Corollary 3.6 is that a horoball is uniquely determined by $x, b$, and $E_{\infty}(F)$. Thus its horofunction is determined by $b$ and $\pi\left(g_{F}\right) \in \Delta_{D \backslash \operatorname{supp}(b)}$. So

$$
\Delta_{\infty}:=\left\{(b, f) \mid b \in \partial \Delta, f \in \Delta_{D \backslash \operatorname{supp}(b)}\right\} .
$$

Remark 3.7. Once one has realized that "directions" are important in the definition of horoballs, one can come up with a more geometric proof of Theorem 3.4.

(1) The case $F=D \backslash \operatorname{supp}(b)$. Here we replace $x$ by $E_{\infty}(F)$ and consider a sequence $\left(g^{n}\right)_{n}$ of points tending along the Euclidean ray issuing from $E_{\infty}(F)$ to $b$. By Example 2.1 this defines an $k$-horoball. According to Theorem 3.4, the shape of the $k$-horoball can be determined by tangential cones as in Lemma 2.3. In other words, we consider the tangential cone at a ball of the sequence in $E_{\infty}(F)$. All further balls are just blow ups of the initial 

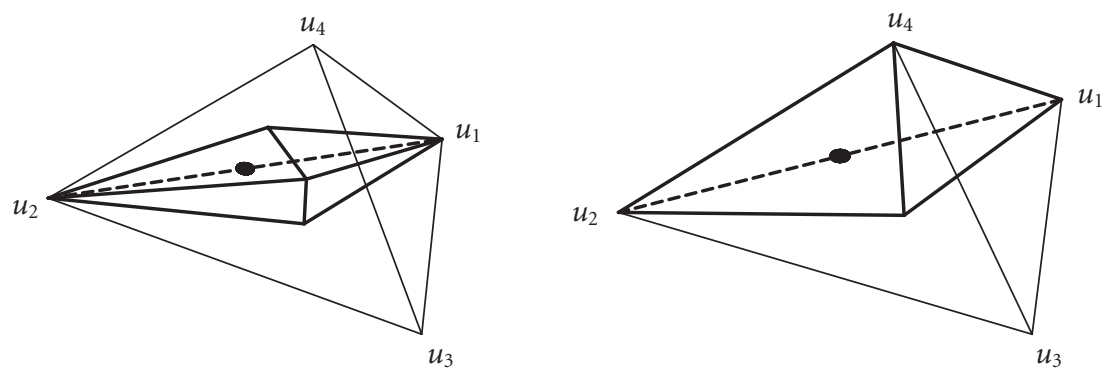

Figure 3.4. Two horoballs centered at $\left(u_{1}+u_{2}\right) / 2$ with $\pi\left(g_{\{3,4\}}\right)$ equal to $\left(u_{3}+u_{4}\right) / 2$ on the left and $u_{4}$ to the right.

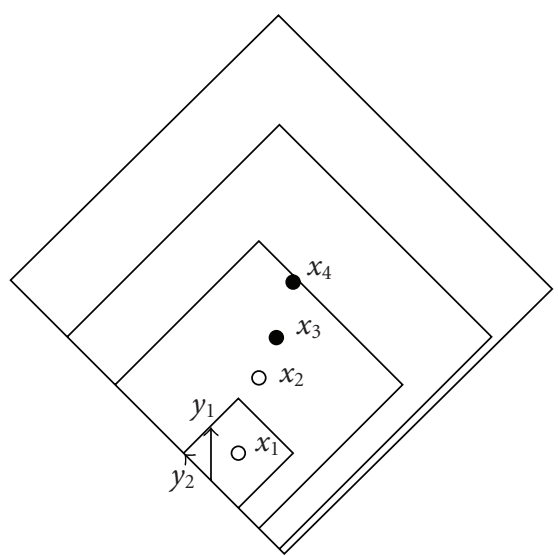

Figure 3.5. Blowing up and shifting a sequence of balls.

one and they exhaust the tangential cone. The resulting horoball coincides with the one of the original sequence. We call this the "blow up" technique.

(2) The case $F=\{i\}$. In a first step, we use (1) for a sequence $\left(g^{n}\right)_{n}$ on a Euclidean ray issuing from $x^{n} \in \Delta$ pointing to $b$ to define a $k$-horoball $B_{n}$. In a second step, we use a sequence $\left(x^{n}\right)_{n} \subset \Delta$ of "directions," tending to a point in $\partial \Delta$ with support $D \backslash\{i\}$. This defines a sequence $\left(B_{n}\right)_{n}$ of $k$-horoballs converging to the "basic" horoball with $F=\{i\}$. Again the resulting horoball equals the original one. This time the horoballs $\left(B_{n}\right)_{n}$ are shifted according to $\left(x^{n}\right)_{n}$. We call this the "shift" technique.

(3) The remaining cases are a mixture of (1) and (2) by Corollary 3.6. We call the resulting geometric construction the "blow up and shift" technique.

The "blow up and shift" technique of Remark 3.7 is used in Figure 3.5 of the next chapter on polyhedral Minkowski spaces.

A consequence of Corollary 3.5 concerns $k$-geodesic rays. It stimulated Foertsch and Karlsson to study the convergence of geodesics more generally with different techniques in [7]. 
COROLLARY 3.8. Every $k$-geodesic ray in $\Delta$ converges to a point $b \in \partial \Delta$.

Proof. Use Remark 3.7 and Corollary 3.5.

\section{Polyhedral Minkowski spaces}

In the case of a general Minkowski space $V$ (i.e., a normed finite dimensional vector space over the reals), the linear ray boundary is only a small part of $V_{\infty}$. Namely, if $\left(x_{n}\right)_{n} \subset V$ is an unbounded sequence tending to $\xi \in V_{\infty}$ and $\mathbb{R}_{+} x_{n}$ tends to $\mathbb{R}_{+} y_{0}$, with $\left|y_{0}\right|=1$, then the ray $\mathbb{R}_{+} y_{0}$ does not determine $\xi$ in general. Additional points of $V_{\infty}$ will occur when $\left(x_{n}\right)_{n}$ "drifts away" from $\mathbb{R}_{+} y_{0}$. Choosing an appropriate subsequence, we will assume that $\left(x_{n}\right)_{n}$ drifts away in the direction of a certain simplicial cone lying in the boundary of the tangent cone $T\left(-y_{0}, B\right)$. This simplicial cone will uniquely determine the limit $\xi \in V_{\infty}$ and the horoballs about $\xi$.

4.1. The norm. Let $B \subset V$ be a bounded, open, centrally symmetric, and convex set. Then $B$ defines a norm $|\cdot|$ on $V$ by

$$
|x|:=\inf \{\alpha>0 \mid x \in \alpha B\} .
$$

The convex set $B$ is termed a polyhedron when it has only finitely many extremal points. The normed space $(V,|\cdot|)$ is then a polyhedral Minkowski space. By definition $B$ is the $|\cdot|$-unit ball. We denote its $|\cdot|$-unit sphere by $S$. Every $v \in S$ defines a ray $\mathbb{R}_{+} v$. This ray is a $|\cdot|$-geodesic ray as we can see from the definition of $|\cdot|$. Thus $(V,|\cdot|)$ is a proper geodesic metric space, and we can use the techniques of Section 2 to define horofunctions and horoballs.

Henceforth we take the origin in $V$ as a reference point. For each $v \in S$ denote by $h_{v}$ the horofunction corresponding to the ray $\mathbb{R}^{+} v$ in the sense that $h_{v}$ is defined by a sequence of points on $\mathbb{R}^{+} v$ tending to infinity.

Lemma 4.1. $B_{v}^{h}(C)=T(-v, B)-C v=T(0, v+B)-C v$.

Proof. The second equality follows from the fact that the tangent cone $T(x, M)$ is invariant under translations of $V$. For the first, use Lemmas 2.2 and 2.3 to deduce

$$
\begin{aligned}
B_{v}^{h}(C) & =d \limsup _{n \rightarrow \infty} B(n v, n+C) \\
& =d \limsup _{n \rightarrow \infty}(B((n+C) v, n+C)-C v) \\
& =T(0, v+B)-C v
\end{aligned}
$$

4.2. Horoballs. Now we can prove the main result of this section.

Theorem 4.2. Let $V=\left(\mathbb{R}^{d},|\cdot|\right)$ be a polyhedral Minkowski space. Let $\left(p_{n}\right)_{n}$ be an unbounded sequence in $V \backslash\{0\}$ which tends to $\xi \in V_{\infty}$, that is,

$$
h_{\xi}(x)=\lim _{n \rightarrow \infty}\left(\left|p_{n}-x\right|-\left|p_{n}\right|\right) .
$$


Then there exist a unit basis $y_{1}, \ldots, y_{d}$ of $V$ and a subsequence $\left(x_{n}\right)_{n}$ of the sequence $\left(p_{n}\right)_{n}$ such that

(1) the cone $\sum_{i=2}^{d} \mathbb{R}_{+} y_{i}$ is contained in $\partial T\left(-y_{1}, B\right)$,

(2) $\lim _{n \rightarrow \infty}\left(x_{n} /\left|x_{n}\right|\right)=y_{1}$,

(3) $x_{n}=\left|x_{n}\right| y_{1}-a_{2 n} y_{2}-\cdots-a_{d n} y_{d}$, for nonnegative $a_{i j}$, and $\lim _{n \rightarrow \infty}\left(a_{i n} /\left|x_{n}\right|\right)=0$ for all $2 \leq i \leq d$.

Furthermore, for any subsequence $\left(n_{k}\right)_{k}$ such that the limits $a_{i}:=\lim _{k \rightarrow \infty} a_{i n_{k}} \in \mathbb{R}_{+} \cup\{\infty\}$ are defined,

$$
B_{\xi}^{h}(C)=T\left(-y_{1}, B\right)-C y_{1}-\sum_{a_{i}=\infty} \mathbb{R}_{+} y_{i}-\sum_{a_{i}<\infty} a_{i} y_{i}
$$

The formula of the horoball in Theorem 4.2 realizes the "blow up and shift" technique of Remark 3.7. The "blow up" is the tangent cone $T\left(-y_{1}, B\right)$, the "shift" can be finite, $-C y_{1}-\sum_{a_{i}<\infty} a_{i} y_{i}$, or infinite, $-\sum_{a_{i}=\infty} \mathbb{R}_{+} y_{i}$, see Figure 3.5.

Proof of Theorem 4.2. Since $B$ is compact, the sequence $\left(p_{n} /\left|p_{n}\right|\right)_{n}$ has an accumulation point $y_{1}$. Let us consider a corresponding subsequence $\left(x_{n}\right)_{n}$. That is, we assume that

$$
\lim _{n \rightarrow \infty} \mathbb{R}_{+} x_{n}=\mathbb{R}_{+} y_{1} .
$$

The boundary $\partial T\left(-y_{1}, B\right)$ of the cone $T\left(-y_{1}, B\right)$ is a union of codimension one (closed) faces $F_{i}, 1 \leq i \leq k$. The vector $y_{1}$ lies in the interior of $T\left(-y_{1}, B\right)$ and hence it belongs to the interior of a larger set $V-\left\{-T\left(-y_{1}, B\right)\right\}$. The last one is the finite union of the cones of the form $\mathbb{R}_{+} y_{1}-F_{i}, 1 \leq i \leq k$. Passing to a subsequence we may assume that $x_{n} \in$ $\mathbb{R}_{+} y_{1}-F$ for some face $F$. Each face $F$ is a finite union of simplicial cones of codimension one. Hence, again passing to subsequence we may assume that $F$ is simplicial, that is, it is spanned by $d-1$ linearly independent unit vectors $y_{2}, \ldots, y_{d}$. Thus each $x_{n}$ has a unique representation, as in Figure 4.1,

$$
x_{n}=a_{1 n} y_{1}-a_{2 n} y_{2}-\cdots-a_{d n} y_{d} .
$$

Because of (4.5), we have $\lim _{n \rightarrow \infty}\left(a_{i n} /\left|x_{n}\right|\right)=0$ for all $2 \leq i \leq d$ and $a_{1 n}=\left|x_{n}\right|$ for all $n$. Thus the sequence $\left(x_{n}\right)$ satisfies the conditions (1)-(3).

To prove the formula for the horoball, we follow Figure 3.5. We notice that

$$
B\left(x_{n},\left|x_{n}\right|+C\right)=B\left(\left|x_{n}\right| y_{1},\left|x_{n}\right|+C\right)-a_{2 n} y_{2}-\cdots-a_{d n} y_{d}
$$

From Lemma 2.2 and equality (4.7), we deduce

$$
\begin{aligned}
B_{\xi}^{h}(C) & =d \limsup _{n \rightarrow \infty} B\left(x_{n},\left|x_{n}\right|+C\right) \\
& =d \limsup _{n \rightarrow \infty}\left(B\left(\left|x_{n}\right| y_{1},\left|x_{n}\right|+C\right)-a_{2 n} y_{2}-\cdots-a_{d n} y_{d}\right) .
\end{aligned}
$$

In a first step we will prove,

$$
d \limsup \left(B\left(\left|x_{n}\right| y_{1},\left|x_{n}\right|\right)-\sum_{I} a_{i n} y_{i}\right)=T\left(-y_{1}, B\right)-\sum_{I} \mathbb{R}_{+} y_{i},
$$


16 Horoballs in simplices and Minkowski spaces

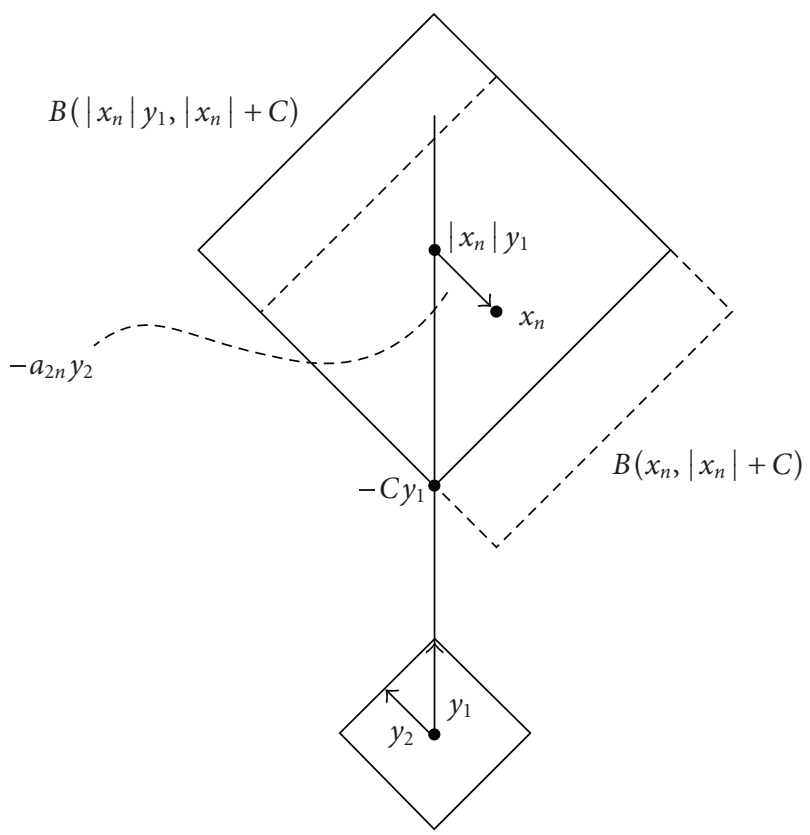

Figure 4.1. The decomposition of $x_{n}$.

where $I$ consists of those $i$, for which $\lim _{n \rightarrow \infty} a_{i n}=\infty$. Let $B_{n}=B\left(\left|x_{n}\right| y_{1},\left|x_{n}\right|\right)$ and recall that by Lemma 4.1,

$$
d \limsup B_{n}=T\left(-y_{1}, B\right)
$$

and moreover, since the sequence of balls above is increasing by inclusion, we have

$$
d \limsup B_{n}=\cup_{n} B_{n}=T\left(-y_{1}, B\right) .
$$

By this $B_{n}-\sum_{I} a_{i n} y_{i}$ is contained in the right-hand side of (4.9). Conversely, suppose that $x$ belongs to the right-hand side. This means that there exist nonnegative $b_{i}-s$ such that

$$
x^{\prime}=x+\sum_{I} b_{i} y_{i} \in T\left(-y_{1}, B\right) .
$$

The latter cone is the union of balls $\cup B_{n}$ by (4.11). Fix a natural number $n_{0}$ such that the "unit cube"

$$
K=\left\{y: y=\sum_{I} c_{i} y_{i}, 0 \leq c_{i} \leq 1, i \in I\right\}
$$

is contained in $B_{n_{0}}$ and $x^{\prime}$ is also contained therein. For $n$ large enough, we have $\lambda_{n}=$ $\left|x_{n}\right| /\left|x_{n_{0}}\right|>1$. By homothety with coefficient $\lambda_{n}$ we obtain

$$
\lambda_{n} K \subset B_{n}, \quad \lambda_{n} x^{\prime} \in B_{n} .
$$


For any $y \in K$ and large enough $n$, the convex combination

$$
\frac{1}{\lambda_{n}} \lambda_{n} x^{\prime}+\left(\frac{\lambda_{n}-1}{\lambda_{n}}\right) \lambda_{n} y=x^{\prime}+\left(\lambda_{n}-1\right) y
$$

belongs to $B_{n}$. It follows that

$$
x+\sum_{I} b_{i} y_{i}+\left(\lambda_{n}-1\right) K \subseteq B_{n}, \quad n \gg 0
$$

In other words, $x+\sum_{I} c_{i} y_{i} \in B_{n}$ if $b_{i} \leq c_{i} \leq b_{i}+\left(\lambda_{n}-1\right)$ for all $i \in I$. To prove that $x$ belongs to the left-hand side of (4.9), it is enough to show that $x+\sum_{I} a_{i n} y_{i} \in B_{n}$ for $n$ sufficiently large. This inclusion would follow from the inequalities $b_{i} \leq a_{\text {in }} \leq b_{i}+\left(\lambda_{n}-\right.$ $1)$. But this is indeed the case for $n \gg 0$ since by assumption the sequences $\left(a_{i n}\right),\left(\lambda_{n}\right)$, $\lambda_{n} / a_{\text {in }}$ each tend to $\infty$ with $n$. This proves the remaining inclusion, that is, (4.9) is verified.

Next, we claim that for any real $C$, we have

$$
d \limsup \left(B\left(\left|x_{n}\right| y_{1},\left|x_{n}\right|+C\right)-\sum_{I} a_{i n} y_{i}\right)=T\left(-y_{1}, B\right)-C y_{1}-\sum_{I} \mathbb{R}_{+} y_{i} \text {. }
$$

Indeed, for $C=0$ this coincides with (4.9). In general, we choose $-C y_{1}$ to be the origin. Although $x_{n}$ is changed to $x_{n}+C y_{1}$, the vectors $y_{1}, y_{2}, \ldots, y_{n}$ and the coefficients $a_{2 n}, \ldots, a_{d n}$ do not change. Hence we obtain the general formula by adding $-C y_{1}$ to the right-hand side.

It remains to add the sum over those $i$ with finite $a_{i}$ on both sides of (4.9). The desired equality follows immediately from the fact that

$$
d \limsup _{n \rightarrow \infty}\left(X_{n}+v_{n}\right)=d \limsup _{n \rightarrow \infty}\left(X_{n}\right)+\lim _{n \rightarrow \infty} v_{n}
$$

for any converging sequence $\left(X_{n}\right)_{n}$ of subsets in $V$ and any converging sequence $\left(v_{n}\right)_{n}$ of points in $V$.

COROLlary 4.3. Under the assumptions of Theorem 4.2 for any $\xi \in V_{\infty}$, there is a geodesic ray $\gamma(t)$ such that

$$
h_{\xi}(t)=\lim _{n \rightarrow \infty}|x-\gamma(t)|-t
$$

In other words, every horofunction is determined by a geodesic ray.

Proof. $h_{\xi}(t)$ is uniquely determined by a sequence, satisfying (1)-(3) of Theorem 4.2, more precisely, by the basis $y_{1}, y_{2}, \ldots, y_{d}$ and by the limits $a_{2}, \ldots, a_{d}$ therein. Now, for fixed $c>0$ consider the ray

$$
\gamma(t)=t y_{1}-\ln (t+c) \sum_{a_{i}=\infty} y_{i}-\sum_{a_{i}<\infty} a_{i} y_{i}, \quad t \geq 0
$$


The sequence $(\gamma(n))$ has the same data as that of $x_{n}$, hence it defines the same horofunction $h_{\xi}$. On the other hand, $\gamma(t)$ is a geodesic ray. Recall the well-known fact that a $C^{1}$-curve in $V$ is geodesic if its velocity vector belongs to the cone spanned by some fixed face of the unit ball at any moment $t$. In our case, for $c$ sufficiently large,

$$
\gamma^{\prime}(t)=y_{1}-\frac{1}{t+c} \sum_{a_{i}=\infty} y_{i}
$$

belongs to the face of $B$, corresponding to the cone $F$ for all $t \geq 0$.

Remark 4.4. This corollary gives a positive answer to Rieffel's paper [17, Question 6.5] in the case of a polyhedral Minkowski space.

\section{Dynamical applications}

Let $(X, d)$ be a metric space. A map $f: X \rightarrow X$ is called ( $d$-)nonexpansive if

$$
d(f(x), f(y)) \leq d(x, y)
$$

for all $x, y \in X$. It is a standard fact that

$$
A:=\lim _{n \rightarrow \infty} \frac{1}{n} d\left(f^{n}(x), x\right)
$$

exists and is independent of $x$. The following theorem was proved in [10].

Theorem 5.1. Let $(X, d)$ be a proper metric space and $f$ a nonexpansive map. Either the orbits of $f$ are bounded, or else for any $x \in X$ there exists a horofunction $h$ with $h(x)=0$ such that

$$
h\left(f^{n}(x)\right) \leq-A n
$$

for every $n \geq 0$.

The conclusion of the theorem immediately implies that, moreover,

$$
\lim _{n \rightarrow \infty}-\frac{1}{n} h\left(f^{n}(x)\right)=A
$$

This general result of course applies in particular to the metric spaces considered in this paper, hence the combination of the description of all horofunctions and Theorem 5.1 give strong information on the dynamics of nonexpansive maps. Such maps arise for Hilbert's metric on convex sets in many important contexts, see, for example, $[4,6,14-$ 16], and so we get from Theorem 5.1 in this setting the following.

Theorem 5.2. Let $\Delta$ be an open simplex and $f: \Delta \rightarrow \Delta$ a $k$-nonexpansive map. For each $x \in \Delta$, either the orbit $f^{n}(x)$ stays in a compact set inside $\Delta$ or there is a horoball with description as in Theorem 3.4 passing through $x$ containing all orbit points $f^{n}(x), n \geq 0$. If

$$
\inf _{x \in \Delta} k(f(x), x)>0
$$

then in addition all accumulation points belong to only one closed face of $\partial \Delta$. 
Proof. The first part of the theorem is just an immediate application of Theorem 5.1, regardless whether the linear rate of escape $A$ is positive or zero.

In the case $(X, d)=(\Delta, k)$ it follows from [13], in view of that $(\Delta, k)$ is isometric to a certain Minkowski space, that $A=\inf _{x \in X} k(f(x), x)$. And when this number is positive, Theorem 5.1 gives us a horofunction $h$ for which

$$
h\left(f^{n}(x)\right) \leq-A n \longrightarrow-\infty .
$$

(Incidentally, we here note that this is stronger than what is obtained in [13] in this finite dimensional setting.) The orbit can now only accumulate at one-closed face in view of the description of all horofunctions in Theorem 3.4.

A similar statement can be formulated for the Minkowski spaces we consider. The closed face in the corollary is the intersection of all horoballs associated with that same distinguished horofunction. We conjectured some time ago that the statement about the limit set being contained in just one closed face should hold without the condition $\inf k(f(x), x)>0$ and we were able to prove that in 2 dimensions and some other special cases, see also [12]. However, Nussbaum has indicated to us that he recently has obtained significant general results on this topic, therefore we leave this issue for now. In any case, our theorem provides some very precise information about the whole orbit, not just the limit set.

Another consequence is the following.

Theorem 5.3. Let $\Delta$ be an open simplex and $f: \Delta \rightarrow \Delta$ a $k$-nonexpansive map. Suppose that the orbits of $f$ converge toward one boundary face which consists of only one point. Then for an associated horofunction, all its horoballs $\mathscr{H}$ are $f$-invariant sets, that is, $f(\mathscr{H}) \subset \mathcal{H}$.

Proof. This follows from the description given in Theorem 3.4 and [10, Theorem 3.4] (actually its proof to be precise).

\section{Acknowledgments}

The authors were supported by the DFG research group "Spektrale Analysis, asymptotische Verteilungen und stochastische Dynamik." The second author was also supported by the START-project Y96-MAT of the Austrian Science Foundation.

\section{References}

[1] W. Ballmann, Lectures on Spaces of Nonpositive Curvature, DMV Seminar, vol. 25, Birkhäuser, Basel, 1995, with an appendix by M. Brin: Ergodicity of Geodesic Flows.

[2] W. Ballmann, M. Gromov, and V. Schroeder, Manifolds of Nonpositive Curvature, Progress in Mathematics, vol. 61, Birkhäuser Boston, Massachusetts, 1985.

[3] G. Beer, Topologies on Closed and Convex Sets, Mathematics and Its Applications, vol. 268, Kluwer Academic, Dordrecht, 1993.

[4] G. Birkhoff, Extensions of Jentzsch's theorem, Transactions of the American Mathematical Society 85 (1957), no. 1, 219-227.

[5] H. Busemann, The Geometry of Geodesics, Academic Press, New York, 1955. 
[6] P. de la Harpe, On Hilbert's metric for simplices, Geometric Group Theory, Vol. 1 (Sussex, 1991), London Mathematical Society Lecture Note Series, Cambridge University Press, Cambridge, 1993, pp. 97-119.

[7] T. Foertsch and A. Karlsson, Hilbert metrics and Minkowski norms, Journal of Geometry 83 (2005), no. 1-2, 22-31.

[8] D. Hilbert, Über die gerade Linie als kürzeste Verbindung zweier Punkte, Mathematische Annalen 46 (1895), no. 1, 91-96.

[9] J.-B. Hiriat-Urruty and C. Lemaréchal, Convex Analysis and Minimization Algorithms I, Grundlehren der Mathematischen Wissenschaften, vol. 305, Springer, Berlin, 1993.

[10] A. Karlsson, Non-expanding maps and Busemann functions, Ergodic Theory and Dynamical Systems 21 (2001), no. 5, 1447-1457.

[11] A. Karlsson and F. Ledrappier, On laws of large numbers for random walks, The Annals of Probability 34 (2006), no. 5.

[12] A. Karlsson and G. A. Noskov, The Hilbert metric and Gromov hyperbolicity, L'Enseignement Mathématique. IIe Série 48 (2002), no. 1-2, 73-89.

[13] E. Kohlberg and A. Neyman, Asymptotic behavior of nonexpansive mappings in normed linear spaces, Israel Journal of Mathematics 38 (1981), no. 4, 269-275.

[14] E. Kohlberg and J. W. Pratt, The contraction mapping approach to the Perron-Frobenius theory: why Hilbert's metric?, Mathematics of Operations Research 7 (1982), no. 2, 198-210.

[15] V. Metz, The short-cut test, Journal of Functional Analysis 220 (2005), no. 1, 118-156.

[16] R. D. Nussbaum, Hilbert's Projective Metric and Iterated Nonlinear Maps, Memoirs of the American Mathematical Society, vol. 75, American Mathematical Society, Rhode Island, 1988.

[17] M. A. Rieffel, Group $C^{*}$-algebras as compact quantum metric spaces, Documenta Mathematica 7 (2002), 605-651.

A. Karlsson: Department of Mathematics, Royal Institute of Technology, Stockholm 10044, Sweden E-mail address: akarl@math.kth.se

V. Metz: Faculty of Mathematics, Bielefeld University, Bielefeld 33501, Germany

E-mail address: metz@mathematik.uni-bielefeld.de

G. A. Noskov: Sobolev Institute of Mathematics, Pevtsova 13, Omsk 644099, Russia

E-mail address: noskov@mathematik.uni-bielefeld.de 


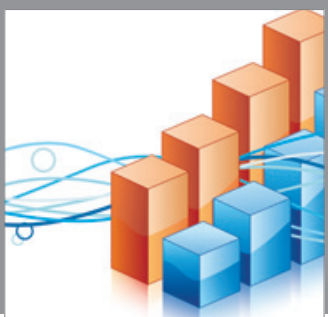

Advances in

Operations Research

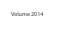

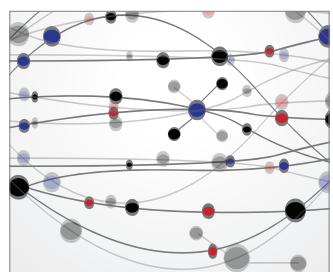

\section{The Scientific} World Journal
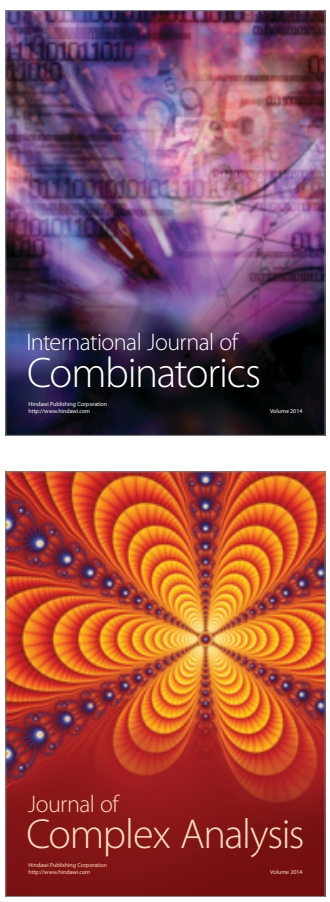

International Journal of

Mathematics and

Mathematical

Sciences
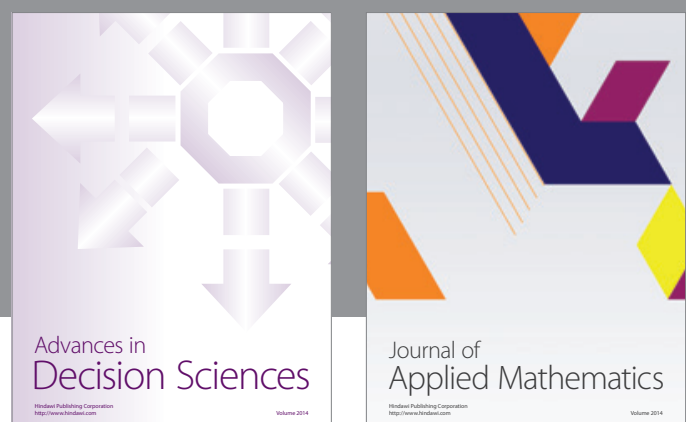

Journal of

Applied Mathematics
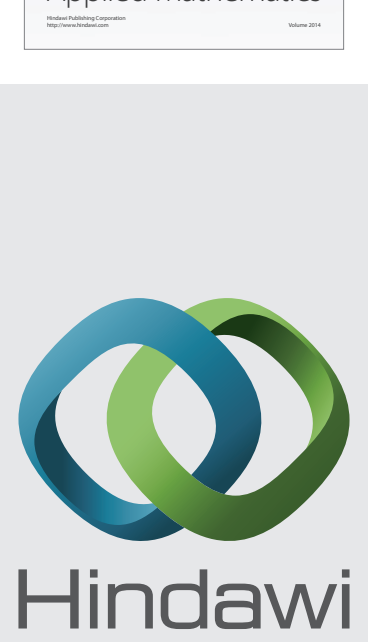

Submit your manuscripts at http://www.hindawi.com
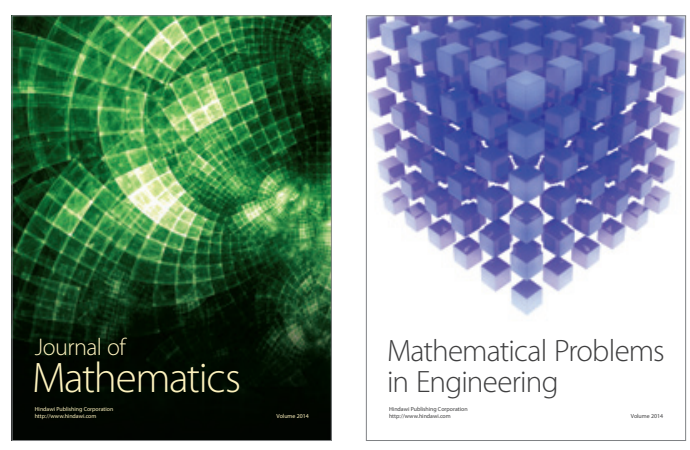

Mathematical Problems in Engineering
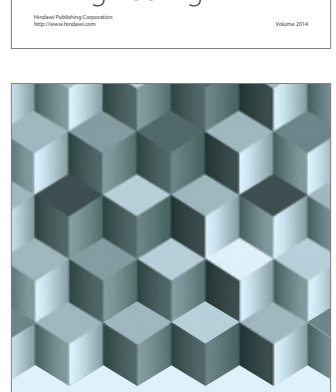

Journal of

Function Spaces
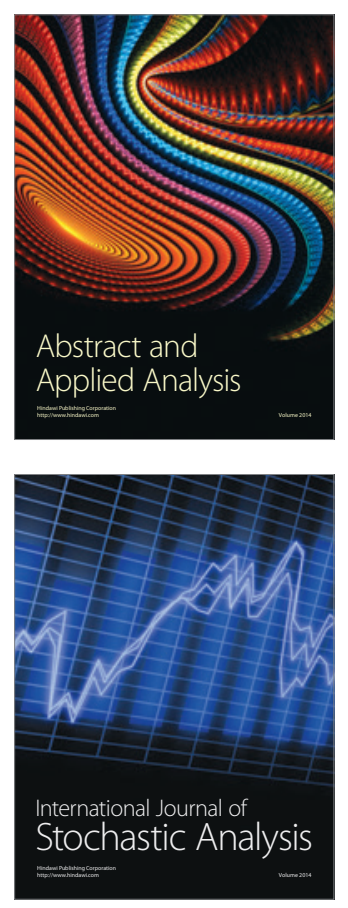

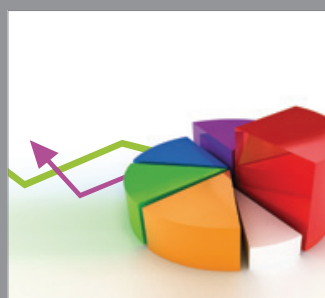

ournal of

Probability and Statistics

Promensencen
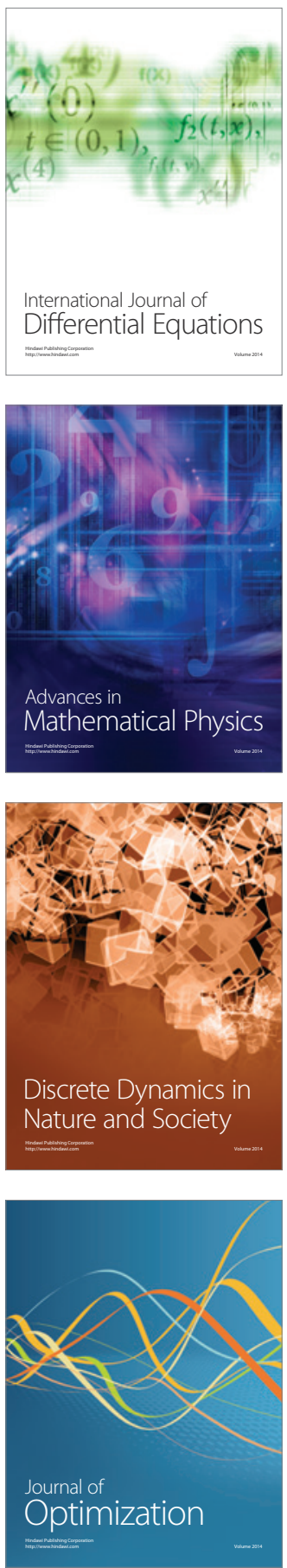